\title{
An Enhanced Distributed Energy-Efficient Clustering (DEEC) Protocol for Wireless Sensor Networks
}

\author{
Amita Yadav $^{1 *}$ and Suresh Kumar ${ }^{2}$ \\ ${ }^{1}$ MSIT, New Delhi \\ ${ }^{2}$ MRIU, Faridabad \\ *amitaay@gmail.com \\ suresh.fet@mriu.edu.in
}

\begin{abstract}
Wireless Sensor Networks (WSNs) is a warm area of research. WSNs consist of large number of sensor nodes deployed randomly in the sensor field. But, it suffers with several shortcomings such as energy imbalance, processing power, storage, transmission range and mitigates energy hole problems. Therefore it's a tough task to design and develop an optimized routing protocol for WSNs. Hence, this research paper mainly focuses on the energy consumption and network lifespan issues of WSNs and presents an improved version of Distributed Energy-Efficient Clustering (DEEC) protocol. To enhance the network lifetime and reliability of DEEC protocol, three improvements are incorporated in the algorithm and the proposed algorithm named as improved DEEC (IDEEC). From experiment section, it is stated that proposed improvements make protocol more robust and efficient in terms of lifetime of nodes, stability and energy consumption in comparison to other algorithm being compared.
\end{abstract}

Keywords: DEEC, LEACH, WSNs and Clustering

\section{Introduction}

For past several years, there is a need for a medium through which entire source of information can be transmitted from the place where it is needed and the received information should be precise to give an absolute and accurate picture of the real world. Hence, a new category of networks has appeared and it is known as wireless sensor networks (WSNs). WSN contains large number of nodes, which collects the information from its surrounding environment. It can also describe as a network of sensing nodes with power facility. These nodes have capability to sense atmospheric or physical parameters such as humidity, temperature, pressure from the surroundings and send all collected information to the central node. These nodes work together to achieve a common goal. In WSN, sensor nodes are used for sensing, processing and communication and it can be either fixed or mobile. There are number of constraint which affect the performance of WSNs, these are energy, processing power, storage and transmission range. Among these, energy is one of main constraint for WSNs. The energy of deployed sensor nodes gradually decreases in terms of data and distance. Lot of research work is going on to address the pitfalls of WSNs. Some of the recent research work in this direction is presented in [1]. In WSNs, sensors are densely deployed for collecting the data and information and it can be widely applicable to different domains such as agriculture, forests, coal mines, monitoring of rail tunnels, monitoring of solar photovoltaic cell in a grid, etc., and collect the data from all locations using a centralized Base Station (BS) with the help of cluster heads (CHs) [2-3]. In such networks, data is collected periodically by the BS.

In Literature, it is found that clustering with hierarchical topology is suitable for continuous monitoring of networks [4]. Further, it is also noticed that clustering improves 
the lifetime of data within network as compared to direct data transmission and it can be improved by a factor of nearly 2 to 3 times [5]. Clustering protocols have proven its advantage in data gathering networks. These protocols also enable data aggregation at cluster head $(\mathrm{CH})$, it decreases transmitted data packets and reduces consumption of energy at sensor nodes [6]. These protocols are communicated in two ways, intra-cluster communication and inter-cluster communication. In WSN, communication can be either single hop or multi-hop [7]. Most of clustering protocols use single hop communication for communicating within clusters such as LEACH [8], LEACH-DT [9], HEED [10], etc. It is also reported that multi-hop communication is more energy-efficient (communication $\mathrm{b} / \mathrm{w}$ sensor node to $\mathrm{CH}$ ) than single hop communication. For example, in dense network, propagation loss exponent is high due to close deployment of sensor nodes and it results in inference and collision problems. Therefore, in such situation, multi-hop communication is more preferable or better than single hop as it successfully overcomes signal propagation difficulties [7]. Another thing in favor of multi-hop is the limitation of direct transmission. For a certain threshold distance, it is preferred to use direct transmission [11] but beyond the threshold, it consumes more energy like to be fourth power of distance [12]. Hence, to improve network scalability, multi-hop communication is more preferable [13]. If $\mathrm{BS}$ is located far away then, it is suggested that to use multihop communication [11]. Some of these are multi-hop LEACH [14], EADC [15] and EDUC [16].

In present scenario, research community focus on the energy imbalance and mitigate energy hole problem of WSNs. A lot of research has been carried out to handle these popular constraints. Hence, the motive of this research work is to improve network lifespan of DEEC protocol [17]. In DEEC, CHs are selected on the basis of probability. It can be computed as the ratio between residual energy of each node and the average energy of network. The main contribution of this research work is as follows-

- $\quad$ To introduce an improved scaling factor parameter to minimize the energy of nodes within cluster.

- $\quad$ To propose a new modified Equation to compute threshold probability more efficiently.

- $\quad$ To extend network life span by using neighborhood information concept.

\section{Related Work}

This section describes related work in the direction of energy efficient protocols especially designed for WSNs. To improve the scalability and energy saving in heterogeneous wireless sensor networks, Heinzelman et al. [8] have developed a clustering based protocol for WSNs known as LEACH protocol. The LEACH was developed to select $\mathrm{CHs}$ randomly for transmission of the energy load from $\mathrm{CHs}$ to BS. It uses the concept of single-hop communication between the sensor nodes and BS. However, the main shortcoming of this protocol is that it is not suitable for large-scale networks. Hence, lot of improvements has been made in LEACH to make it more efficient and powerful, such as LEACH-DT [9], M-LEACH [1].Younis and Fahmy [10] have introduced a distributed energy efficient protocol for Ad-hoc sensor networks, known as HEED protocol. In this protocol, residual energy is used to elect the $\mathrm{CHs}$ and selection of these $\mathrm{CHs}$ is done periodically. This protocol uses multi-hop communication. The performance of the HEED protocol is compared with LEACH protocol and claimed that HEED protocol provides better results in terms of network lifetime. To reduce the energy consumption and to improve the scalability and lifetime of the network, Qing et al. [17], have presented another protocol for WSNs known as DEEC. In this protocol, CHs are selected based on residual energy and average energy of the network. It is also stated that simulation results of DEEC is more significant than any other algorithms being compared with. To prolong the network life time, Ye et al. [18] have presented an energy 
efficient protocol called as EECH. In this algorithm $\mathrm{CHs}$ are selected by using the localized competition. Simulation results of the protocol compared with LEACH and HEED it was observed that the EECH protocol consumes energy more efficiently and significantly improves the lifetime. To improve network lifetime and reliability, Kumar et al. [19] have introduced an improved version of EECH. In this protocol, $\mathrm{CHs}$ are elected on the behalf of weighted probabilities of initial energy nodes. The results of the proposed protocol are compared with LEACH and EECH protocols. The author claimed that the protocol significantly handle the issues of Network life time and reliability. In continuation of their work, Kumar et al. [20] have developed another protocol to address the issues of network lifetime and energy efficiency known as EECDA. This protocol is based on the concept of energy efficient clustering routing and data aggregation. A new $\mathrm{CHs}$ selection and data communication mechanism were adopted for this algorithm. The experimental results were compared with LEACH, EECH and EDGA. From results, it is stated that EECDA protocol achieves better results both in terms of network lifetime and energy efficiency.

\section{Distributed Energy- Efficient Clustering (DEEC) Algorithm}

DEEC is a well-known energy efficient protocol for heterogeneous WSNs. In DEEC, the field is divided into different clusters. Each cluster contains some sensor nodes and has a $\mathrm{CH}$. The work of the $\mathrm{CH}$ is to receive the information from sensor nodes within a cluster and send this information to BS. To make the node as Cluster Head $(\mathrm{CH})$, a probability function is computed. This function is defined in terms of residual energy and average energy of networks. This function computes the ratio between residual energy of each node and the average energy of the network and it is computed for each node of a cluster. The node having high computation value in comparison to other nodes have better chance to be selected as a $\mathrm{CH}$. In WSN, CHs are chosen periodically. A cluster head selection algorithm is adopted for this. The main work of $\mathrm{CH}$ is to collect the data within a cluster and send it to BS. It is also assumed that all nodes of WSN having different amount of energy initially and newly added nodes or energy-harvested nodes have more energy than the old ones. It is also noted that probability of $\mathrm{CHs}$ are also influenced by the ratio between residual energy of each node and the average energy of the network.

\subsection{Heterogeneous Network Model}

A network model of $\mathrm{M} \times \mathrm{M}(100 \times 100)$ square region is considered for experimental purpose. It is noted that ' $n$ ' number of stationary nodes are uniformly dispersed in abovementioned region. The BS is located in the center of square region and nodes consist of data, which is sent to the BS. Among these nodes, some nodes are selected as CHs and $\mathrm{CHs}$ are responsible to transmit the aggregated data to BS. The sensor nodes are categorized into two types-advanced nodes and normal nodes. The initial energy of normal nodes is denoted by $E_{o}$ and ' $m$ ' denotes the fraction of advanced nodes. So in a WSN total advanced nodes are $\mathrm{mN}$ and the energy associated with these nodes is $\mathrm{E}_{\mathrm{o}}$ $(1+a)$. Whereas, total number of normal nodes is $(1-\mathrm{m}) \mathrm{N}$ and the energy associated with these nodes is $E_{0}$. Hence, the total initial energy of the two-level heterogeneous networks is computed using Equation 1.

$$
\mathrm{E}_{\text {total }}=\mathrm{N}(1-\mathrm{m}) \mathrm{E}_{0}+\mathrm{NmE}_{0}(1+\mathrm{a})=\mathrm{NE}_{0}(1+\mathrm{am})
$$

\subsection{Cluster Heads Selection Algorithm}

This subsection describes the process of $\mathrm{CHs}$ selection. Assume the number of nodes disseminated in the region is " $\mathrm{S}$ ". A probability function is associated with each node in each round whether a node is elected as $\mathrm{CH}$ or not. The value of $n_{i}$ differs in each node 
because every node has different energy associated with it. Hence, the probability of a node to be a $\mathrm{CH}$ after $n_{\mathrm{i}}$ rounds can be calculated using Equation 2 .

$$
\mathrm{P}_{\mathrm{i}}=\frac{1}{\mathrm{n}_{\mathrm{i}}}
$$

If nodes consist of same energy level in each round then $\mathrm{P}_{\mathrm{i}}=\mathrm{P}_{\text {opt }}$. If nodes consists of different amount of energy, then the value of $\mathrm{P}_{\mathrm{i}}$ is larger for the nodes having more energy in comparison to nodes with low energy i.e. $\mathrm{P}_{\mathrm{i}}>\mathrm{P}_{\text {opt. }}$ Then the average energy of network can be computed using Equation 3.

$$
\bar{E}(r)=\frac{1}{N} \sum_{i=1}^{N} E_{i}(r)
$$

Using it as reference energy, probability of ith node can be described using Equation 4 and the average number of clusters per epoch is identified using Equation 5.

$$
\begin{aligned}
& P_{i}=P_{o p t}\left[1-\frac{\bar{E}(r)-E_{i}(r)}{\bar{E}(r)}\right]=P_{o p t} \frac{E_{i}(r)}{\bar{E}(r)} \\
& \sum_{i=1}^{N} P_{i}=\sum_{i=1}^{N} P_{o p t} \frac{E_{i}(r)}{\bar{E}(r)}=P_{o p t} \sum_{i=1}^{N} \frac{E_{i}(r)}{\bar{E}(r)}=P_{o p t} N
\end{aligned}
$$

The threshold probability $\mathrm{T}\left(\mathrm{s}_{\mathrm{i}}\right)$ decides whether $\mathrm{S}_{\mathrm{i}}$ can become a $\mathrm{CH}$ or not, is computed using Equation 6.

$$
\mathrm{T}\left(\mathrm{s}_{\mathrm{i}}\right)= \begin{cases}\frac{\mathrm{P}_{\mathrm{i}}}{1-\mathrm{P}_{\mathrm{i}}\left(\mathrm{r} \bmod \frac{1}{\mathrm{P}_{\mathrm{i}}}\right)} & \text { if } \mathrm{S}_{\mathrm{i}} \in \mathrm{G} \\ 0 & \text { otherwise }\end{cases}
$$

In each round, if $\mathrm{S}_{\mathrm{i}}$ finds itself eligible for $\mathrm{CH}$, a random number is selected in between 0 to 1 . The selected number is compared with threshold value, if it is less, then the node acts as $\mathrm{CH}$ in that particular round. $\mathrm{n}_{\mathrm{i}}$ is inverse of $\mathrm{P}_{\mathrm{i}}$ and it is chosen on the basis of residual energy $E_{i}(r)$ which is computed using Equation 7.

$$
n_{i}=\frac{1}{p_{i}}=\frac{\bar{E}(r)}{P_{o p t} E_{i}(r)}=n_{o p t} \frac{\bar{E}(r)}{E_{i}(r)}
$$

According to Equation 7, nodes with high residual energy have more chance to be elected as a $\mathrm{CH}$. It also observes that at every epoch every node is busy in checking its candidature for cluster head. The energy dissipation can be minimized by reducing the number of calculations and it can be possible only by restricting the number of nodes. The nodes having more energy than a threshold value are considered to participate. Hence, a node $S_{i}$ can compete for cluster head selection if $E_{i}(r)>E_{\text {threshold. }}$ In WSNs, initially the nodes have different amount of initial energy. The weighted probability for advance $\left(\mathrm{P}_{\mathrm{adv}}\right)$ and normal $\left(\mathrm{P}_{\mathrm{nrm}}\right)$ is computed using Equation 8.

$$
\mathrm{P}_{\mathrm{adv}}=\frac{\mathrm{P}_{\mathrm{opt}}}{1+\alpha \mathrm{m}}, \mathrm{P}_{\mathrm{nrm}}=\frac{\mathrm{P}_{\mathrm{opt}}(1+\alpha)}{1+\alpha \mathrm{m}}
$$

Thus the $\mathrm{P}_{\mathrm{i}}$ can be described using Equation 9 .

$$
P_{i}= \begin{cases}\frac{P_{o p t} E_{i}(r)}{(1+\alpha m) \bar{E}(r)} & \text { if } S_{i} \text { is the normal node } \\ \frac{P_{\text {opt }}(1+\alpha) E_{i}(r)}{(1+\alpha m) \bar{E}(r)} & \text { if } S_{i} \text { is advanced node }\end{cases}
$$

Further, it can extend to multi-level heterogeneous networks and the weighted probability is calculated using Equation 10, whereas, Pi can be computed using Equation 11.

$$
\mathrm{P}\left(\mathrm{s}_{\mathrm{i}}\right)=\frac{\mathrm{P}_{\mathrm{opt}} \mathrm{N}\left(1+\alpha_{\mathrm{i}}\right)}{\left(\mathrm{N}+\sum_{\mathrm{i}+1}^{\mathrm{N}} \alpha_{\mathrm{i}}\right)}
$$




$$
P_{i}=\left(\frac{P_{o p t} N(1+\alpha) E_{i}(r)}{\left(N+\sum_{i=1}^{N} \alpha_{i}\right) \bar{E}(r)}\right)
$$

\subsection{Energy Consumption}

In DEEC, all nodes share the information about total energy and life time of network. The average probability $\left(\mathrm{P}_{\mathrm{i}}\right)$ is computed using average energy $\left.\overline{(\mathrm{E}}(\mathrm{r})\right)$ and total energy $\left(\mathrm{E}_{\text {total }}\right)$ of network. The life time value $(\mathrm{R})$ is given by the BS. The average energy of network is calculated using Equation 12.

$$
\bar{E}(r)=\frac{1}{N} E_{\text {total }}\left(1-\frac{r}{R}\right)
$$

Here, R represents the network life time, and it can be computed using Equation 13.

$$
\mathrm{R}=\frac{\mathrm{E}_{\text {total }}}{\mathrm{E}_{\text {round }}}
$$

The energy expansion is computed using Equation 14 and it is defined in terms of radio transmission of l-bit message and distance (d).

$$
E_{T x}(l, d)= \begin{cases}l E_{\text {elec }}+l \epsilon_{f s} d^{2}, & d<d_{0} \\ l E_{\text {elec }}+l \epsilon_{m p} d^{4} & d \geq d_{0}\end{cases}
$$

Where $\mathrm{E}_{\text {elec }}$ denotes energy dissipated to run the transmitter on the receiver circuit, and $\epsilon_{\mathrm{fs}} \mathrm{d}^{2}$ and $\epsilon_{\mathrm{mp}} \mathrm{d}^{4}$ is energy dissipated in free environment and multipath path loss. Each node sends $\mathrm{L}$ bits of data to $\mathrm{CH}$ in a single iteration. Hence, energy dissipated in a network is computed using Equation 15.

$$
\mathrm{E}_{\text {round }}=\mathrm{L}\left(2 \mathrm{NE}_{\mathrm{elec}}+\mathrm{NE}_{\mathrm{DA}}+\mathrm{k} \epsilon_{\mathrm{mp}} \mathrm{d}_{\mathrm{CHtoBS}}^{4}+\mathrm{N}_{\mathrm{fs}} \mathrm{d}_{\mathrm{NtoCH}}^{2}\right)
$$

Where, $\mathrm{k}$ defines no. of clusters, $\mathrm{E}_{\mathrm{DA}}$ denotes data aggregation cost, $\mathrm{d}_{\mathrm{CHtoBS}}$ denotes average distance of $\mathrm{CH}$ and $\mathrm{BS}$ and $\mathrm{d}_{\mathrm{NtoCH}}$ is the average distance of nodes and $\mathrm{CH}$. $\mathrm{d}_{\mathrm{CHtoBS}}$ and $\mathrm{d}_{\mathrm{NtoCH}}$ is computed using Equation 16.

$$
\mathrm{d}_{\mathrm{toCH}}=\frac{\mathrm{M}}{\sqrt{2 \pi \mathrm{k}}}, \quad \mathrm{d}_{\mathrm{toBS}}=0.765 \frac{\mathrm{M}}{2}
$$

\section{Proposed Improvements in DEEC Algorithm}

This section describes the proposed improvements in the DEEC algorithm. In this work, three improvements are incorporated in DEEC protocol for enhancing its performance in terms of energy and packet lifetime. These improvements are discussed as follows.

\subsection{Scaling Factor}

To minimize the energy of nodes within a cluster, a scaling factor parameter is introduced. It assumes that the nodes are capable of directly communicating to the base station located a farther distance, thus when intra-cluster communication is to be conducted it should be done at a reduced power level. For example if the network field is $100 \times 100$ in size and there are 10 clusters this means the size of one cluster is $10 \times 10$, thus the maximum power of intra cluster communication may be limited to a little more than $10 \times 10$ size. In other words the amplification power $\epsilon_{\mathrm{fs}}$ could be reduced by a factor of 10. Thus the scaling factor can be defined using Equation 17.

$$
\text { Scaling factor }=\left\{\operatorname{rand}() \times \frac{\text { Area of Network field }}{\text { area of the cluster } \times \text { no. of nodes in a cluster }}\right\}
$$

\subsection{Modified Threshold Probability}

The threshold probability has significant impact on the selection of CHs. It determines whether a node can become a $\mathrm{CH}$ or not. A node can be acted, as a $\mathrm{CH}$ after nth epoch, is calculated using Equation 2. This Equation is used to determine number of nodes live 
after nth epoch, but it cannot consider the energy of nodes that are live after nth epoch. Hence, a new modified Equation is proposed to determine the probability, whether a node becomes a $\mathrm{CH}$ or not which is described as follows.

$$
P_{i}=\frac{\text { Energy of the ith node }}{\sum_{i=1}^{n} \text { Total energy of all nodes within a cluster }}
$$

After computing the probability of $\mathrm{i}^{\text {th }}$ node, it is the threshold probability, which recognize whether a node is capable to become $\mathrm{CH}$ or not. Hence, to improve the efficiency of the DEEC algorithm for CHs selection, total energy of all nodes within a cluster is considered for computing threshold probability and it can be defined using Equation 19.

$$
\mathrm{T}\left(\mathrm{s}_{\mathrm{i}}\right)= \begin{cases}\frac{\mathrm{P}_{\mathrm{i}}}{1-\mathrm{P}_{\mathrm{i}}\left(\mathrm{rmod} \frac{\mathrm{P}_{\mathrm{i}}}{\sum_{i=1}^{n} \mathrm{P}_{\mathrm{i}}}\right)} & \text { if } \mathrm{S}_{\mathrm{i}} \in \mathrm{G} \\ 0 & \text { otherwise }\end{cases}
$$

\subsection{Neighborhood Information}

The concept of neighborhood information is used to identify the next possible CHs. Therefore to compute the location of next $\mathrm{CH}$, a distance-based measure is applied on the current $\mathrm{CH}$ location. So a threshold distance $\left(\mathrm{d}_{\mathrm{o}}\right)$ is taken into account to find the neighbors of current $\mathrm{CH}$. If energy of nodes within threshold distance is less than current $\mathrm{CH}$ energy, all these nodes are rejected, otherwise nodes are selected as neighbor of current $\mathrm{CH}$ and $\mathrm{CH}$ stores all its neighbors. The objective of neighborhood information is to choose a $\mathrm{CH}$ with maximum energy among its neighbors. Hence, the nodes having greater energy than its $\mathrm{CH}$ become neighbors of current $\mathrm{CH}$.

\section{Experimental Results}

This section describes the experimental results of proposed protocol. The proposed algorithm is implemented in MATLAB 2014 using corei5 processor and 4 GB RAM on window operating system. The performance parameters to test the effectiveness and efficiency of proposed protocol are stability period, lifetime and data packets. The results are also compared with other well-known existing algorithms like LEACH and DEEC. In our experiment, the WSNs consist of hundred nodes $(\mathrm{N})$. The nodes are randomly scattered in $100 \mathrm{~m} \times 100 \mathrm{~m}$ dimension field. It is assumed that all nodes are stationary. It is also considered that BS is placed at center of network field. For heterogeneous WSNs, the settings of radio parameters are shown in Table 1. The uniform distribution of sensor nodes in sensor filed is shown in Figure1, where circles represent the sensor nodes, whereas star represents the centrally located base station.

Table 1. Parameter Setting of IDEEC Protocol

\begin{tabular}{|c|c|c|c|}
\hline Parameter & Value & Parameter & Value \\
\hline Network field & $100 \mathrm{~m} \times 100 \mathrm{~m}$ & $\mathrm{E}_{\mathrm{DA}}$ & $5 \mathrm{~nJ} / \mathrm{bit} /$ Message \\
\hline Number of nodes & 100 & $\mathrm{~d}_{0}$ & $70 \mathrm{~m}$ \\
\hline $\mathbf{E}_{\text {elec }}$ & $5 \mathrm{~nJ} / \mathrm{bit}$ & Message Size & $4000 \mathrm{bits}$ \\
\hline $\mathbf{E}_{\mathrm{fs}}$ & $10 \mathrm{pJ} / \mathrm{bit} / \mathrm{m}^{2}$ & $\mathrm{P}_{\mathrm{opt}}$ & 0.1 \\
\hline $\mathbf{E}_{\mathrm{amp}}$ & $0.0013 \mathrm{pJ} / \mathrm{bit} / \mathrm{m}^{2}$ & $\mathrm{E}_{\text {Threshold }}$ & $\mathrm{Eo} / 4$ \\
\hline $\mathbf{E}_{\mathbf{0}}$ & $0.5 \mathrm{~J}$ & Scale Factor & 10 \\
\hline
\end{tabular}




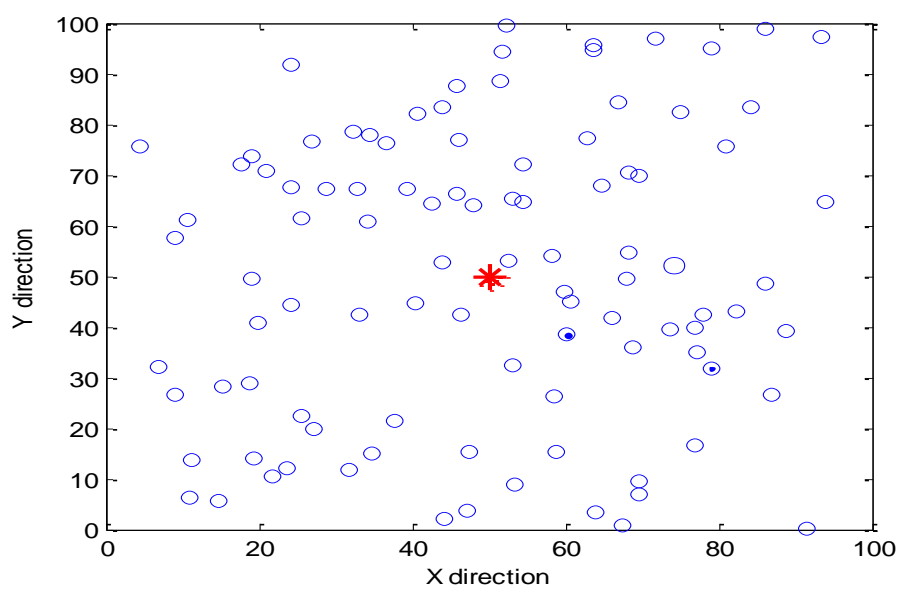

Figure 1. Uniform Distribution of Sensor Node in $100 \mathrm{~m} \times 100 \mathrm{~m}$ Sensor Field with BS

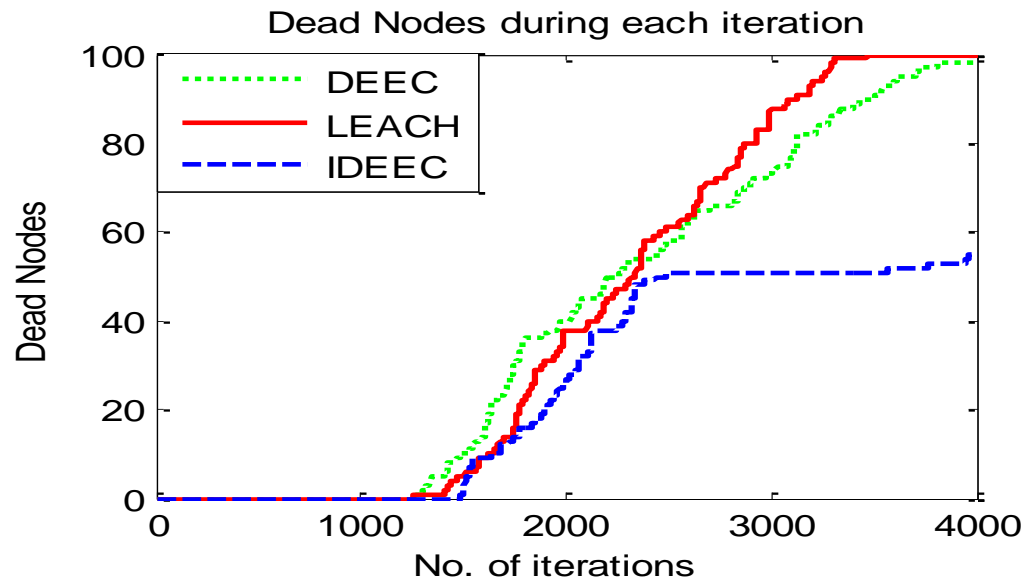

Figure 2. Comparison of Dead Nodes of IDEEC, DEEC and LEACH Protocols

Figure 2 show the comparison of dead nodes in each iteration of IDEEC, DEEC and LEACH protocols over 4000 iterations. In first 1000 iterations, all nodes are live nodes. In DEEC and LEACH protocols, after 1200 iteration nodes become dead as entire energy is consumed. Whereas, in proposed IDEEC algorithm nodes become dead after 1500 iterations. In proposed IDEEC protocol, only 48 nodes are converted into dead nodes throughout its entire iterations. Whereas, in LEACH all nodes become dead nearly after 3100 iterations and in DEEC, only 8 nodes are live after 4000 iterations. From this, it can be concluded that the proposed protocol is more effective and efficient. Hence the proposed improvements make the DEEC protocol prolong in the direction of network lifetime. Figure 3 shows the comparison of packets sent to the BS using IDEEC, DEEC and LEACH protocols. From Figure 3, it is clearly observed that there is significant difference between the packets sent by IDEEC protocol and other protocol being compared. 


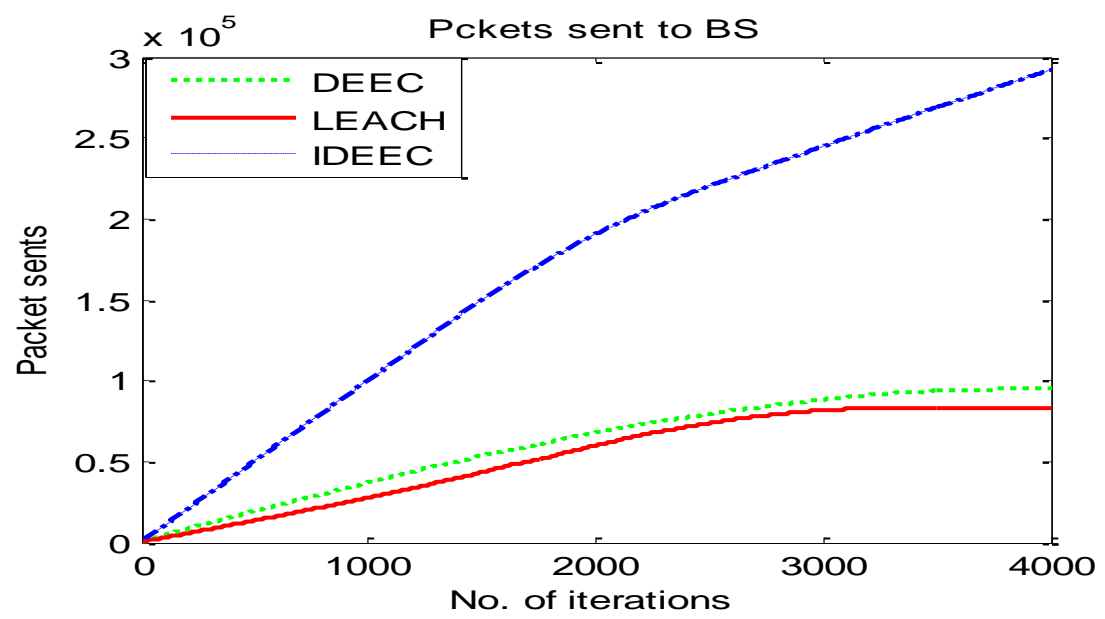

Figure 3. Comparison of Packet Sent to BS of IDEEC, DEEC and LEACH Protocols

\section{Conclusion}

In this work, three improvements are integrated in DEEC protocol to make it more effective, efficient and stable for WSNs. Basically DEEC is adaptive energy efficient protocol to design the change in probability of sensor nodes for becoming a $\mathrm{CH}$. To make the protocol more robust, capable and reliable, few modifications are proposed in this work. These modifications target the packet lifetime and energy consumption issues of DEEC. Hence, the concept of scaling parameter and neighborhood information are integrated in existing DEEC protocol. Among these, a modified threshold probability Equation is also proposed to select the $\mathrm{CH}$ in efficient way. The experimental results of the proposed protocol are compared with LEACH and DEEC protocols. Among these protocols, proposed protocol gives better and more efficient results.

\section{References}

[1] V. Mhatre and C. Rosenberg, "Homogeneous vs. heterogeneous clustered networks: a comparative study”, Proceedings IEEE, ICC, vol. 6, (2004), pp. 3646-3651.

[2] P. Ayona and A. Rajesh, "Investigation of energy efficient sensor node placement in railway systems", Eng. Sci. Technol. International Journal, (2015), doi:10.1016/j.jestch.2015.10.009.

[3] V. Kaundal, A. K. Mondal, P. Sharma and K. Bansal, "Tracing of shading effect on underachieving SPV cell of an SPV grid using wireless sensor network", Eng. Sci. Technol. International Journal, vol. 18, (2015), pp. 475-484.

[4] S. Bandyopadhyay and E. J. Coyle, "An energy efficient hierarchical clustering algorithm for wireless sensor networks", IEEE INFOCOM, (2003), pp. 1713- 1723.

[5] A. F. Liu, W. X. You, C. Z. Gang and G. W. Hua, "Research on the energy hole problem based on unequal cluster-radius for wireless sensor networks", Comp. Commun., vol. 33, (2010), pp. 302-321.

[6] M. Liu, J. Cao, G. Chen and X. Wang, "An energy aware routing protocol in wireless sensor networks", Sensors, vol. 9, (2009), pp. 445-462.

[7] V. Mhatre and C. Rosenberg, "Design guidelines for wireless sensor networks: communication", clustering and aggregation, Ad Hoc Netw., vol. 2, no. 1, (2004), pp. 45-63.

[8] W. B. Heinzelman, A. Chandrakasan and H. Balakrishnan, "Energy efficient communication protocol for wireless microsensor networks", Proceedings 33rd Hawaii Intl Conference on System Sciences (HICSS'00), (2000), pp. 8020-8029.

[9] S. H. Kang and T. Nguyen, "Distance based thresholds for cluster head selection in wireless sensor networks", IEEE Commun. Lett., vol. 16, no. 9, (2012), pp. 1396-1399.

[10] O. Younis and S. Fahmy, "HEED: a hybrid, energy-efficient, distributed clustering approach for ad hoc sensor networks", IEEE Trans. Mobile Comput., vol. 3, no. 4, (2004), pp. 366-379.

[11] B. Tavli, "Energy-efficient relaying in wireless networks", International Journal Electron.Commun., vol. 63, (2009), pp. 695-698. 
[12] W. B. Heinzelman, A. Chandrakasan and H. Balakrishnan, "An application-specific protocol architecture for wireless microsensor networks", IEEE Trans. Wirel.Commun., vol. 1, no. 4, (2002), pp. 660-670.

[13] R. C. Carrano, D. Passons, L. C. S. Maglhaes and V. N. Albuquerque, "Survey and taxanomy of duty cycling mechanisms in wireless sensor networks", IEEE Commun. Surv. Tut., vol. 16, no. 1, (2014), pp. 181-192.

[14] F. Xiangning and S. Yulin, "Improvement on LEACH protocol of wireless sensor network", Proceedings of International Conference on Sensor Technologies and Applications (Sensor Comm), (2007), pp. 260-264, doi:10.1109/SENSORCOMM.2007.4394931.

[15] J. Yu, Y. Qi, G. Wang and X. Gu, "A cluster-based routing protocol for wireless sensor networks with non-uniform node distribution", International Journal Electron. Commun., vol. 66, (2012), pp. 54-61.

[16] J. Yu, Y. Qi and G. Wang, "An energy driven unequal clustering protocol for heterogeneous wireless sensor networks", Journal Control Theory Appl., vol. 9, no. 1, (2011), pp. 133-139.

[17] L. Qing, Q. Zhu and M. Wang, "Design of a distributed energy-efficient clustering algorithm for heterogeneous wireless sensor networks", Computer communications, vol. 29, no. 12, (2006), pp. 22302237.

[18] M. Ye, C. Li, G. Chen and J. Wu, "EECS: an energy efficient cluster scheme in wireless sensor networks", IEEE International Workshop on Strategies for Energy Efficiency in Ad Hoc and Sensor Networks (IEEE IWSEEASN-2005), Phoenix, Arizona, (2005).

[19] D. Kumar, T. C. Aseri and R. B. Patel, "EEHC: Energy efficient heterogeneous clustered scheme for wireless sensor networks", International Journal of Computer Communications, Elsevier, vol. 32, no. 4, (2009), pp. 662-667.

[20] D. Kumar, T. C. Aseri and R. B. Patel, "EECDA: energy efficient clustering and data aggregation protocol for heterogeneous wireless sensor networks", International Journal of Computers Communications \& Control, vol. 6, no. 1, (2011), pp. 113-124.

\section{Authors}

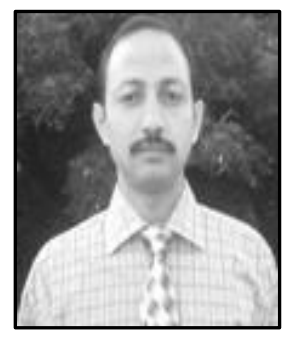

Suresh Kumar, he currently working as a Professor in CSE Department at Manav Rachna International University, Faridabad. $\mathrm{He}$ is a life member of ISTE and CSI. He is also a member of IEEE and IACSIT. He is working in the area of Adhoc Networks, Information retrieval, Big Data Analytics, and Databases. He has published more than forty research papers in International Journals and Conferences, including IEEE, ACM and Springer etc. He is actively involved in research activities and is on the reviewing panel of many journals and conferences.

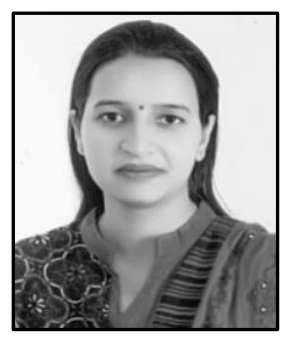

Amita Yadav, she received her Master's degree in Computer science \& engineering from G.J.U, Hisar. She is currently a doctoral candidate in Manav Rachna international university. Her research interest is mainly in the area of Wireless Networks, Network Security and Data mining. 
International Journal of Future Generation Communication and Networking Vol. 9, No. 11 (2016) 